IMAGES IN MEDICINE

\title{
Renal and extrarenal autosomal dominant polycystic kidney disease
}

A 50 year old man with no past medical history presented with right upper quadrant abdominal pain and a history of occasional headaches. Physical examination was remarkable for an enlarged, nodular non-tender liver which was palpable $8 \mathrm{~cm}$ below the costal margin. Computed tomography of the abdomen revealed multiple cysts in the liver and in both the kidneys (fig 1). An isolated cyst was also visualised in the pancreas (arrowed). The patient was adopted and therefore family history was not available. His kidney and liver function tests were normal. As he reported occasional headaches, magnetic resonance angiography (MRA) of the brain was performed. A small berry aneurysm of the anterior communicating artery was visualised (fig 2, arrow). The patient was referred for neurosurgical evaluation. Our patient had features consistent with autosomal dominant polycystic kidney disease (ADPKD) which affects 500000 people in the USA. Extrarenal manifestations include hepatic cysts $(50 \%)$, pancreatic cysts $(10 \%)$, and cerebral aneurysms $(10 \%)$. Patients with ADPKD have a higher incidence of subarachnoid haemorrhage because of rupture of these cerebral aneurysms. Patients with cerebral symptoms should be screened for aneurysms, initially with MRA, and then if necessary with real time angiography.

SHEHZAD BASARIA NIRAJ N MEHTA

Department of Internal Medicine, The University of Texas Health Science Center at Houston, LBF Hospital, Houston, Texas, USA

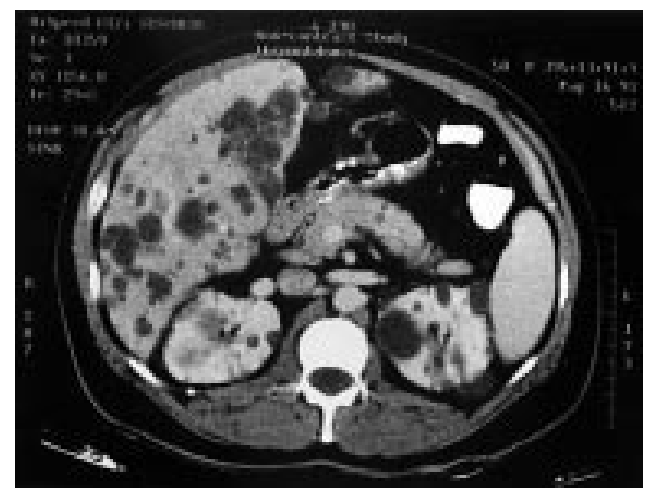

Figure 1 Computed tomography of the abdomen showing multiple cysts in the liver, both kidneys, and pancreas.

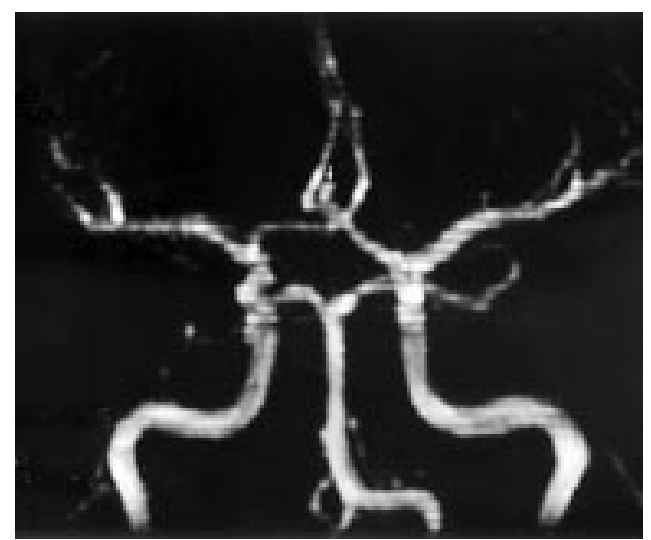

Figure 2 Magnetic resonance angiography of the brain, showing a small berry aneurysm of the anterior communicating artery. 